\section{Quantification of minimal disseminated disease by quantitative polymerase chain reaction and digital polymerase chain reaction for NPM-ALK as a prognostic factor in children with anaplastic large cell lymphoma}

\author{
Christine Damm-Welk, ${ }^{1}$ Nina Kutscher, ${ }^{1}$ Martin Zimmermann, ${ }^{2}$ Andishe \\ Attarbaschi, ${ }^{3}$ Jutta Schieferstein, ${ }^{1}$ Fabian Knörr, ${ }^{4}$ Ilske Oschlies, ${ }^{5}$ Wolfram \\ Klapper ${ }^{5}$ and Wilhelm Woessmann $n^{1,4}$ \\ ${ }^{1}$ NHL-BFM Study Center, Department of Pediatric Hematology and Oncology, Giessen, \\ Germany; ' Department of Pediatric Hematology and Oncology, Hannover Medical School, \\ Hannover, Germany; ${ }^{3}$ Department of Pediatric Hematology and Oncology, St. Anna \\ Children's Hospital, Medical University of Vienna, Vienna, Austria; ${ }^{4}$ Pediatric Hematology \\ and Oncology, University Medical Center Hamburg-Eppendorf, Hamburg, Germany and \\ ${ }^{5}$ Institute of Pathology, Hematopathology Section and Lymph Node Registry, Kiel, \\ Germany.
}

- Present address: Pediatric Hematology and Oncology, University Medical Center Hamburg-Eppendorf, Hamburg, Germany

\section{ABSTRACT}

$\mathrm{D}$ etection of minimal disseminated disease is a validated prognostic factor in ALK-positive anaplastic large cell lymphoma. We previously reported that quantification of minimal disease by quantitative real-time polymerase chain reaction (RQ-PCR) in bone marrow applying a cut-off of 10 copies $N P M-A L K / 10^{4}$ copies of $A B L 1$ identifies very high-risk patients. In the present study, we aimed to confirm the prognostic value of quantitative minimal disseminated disease evaluation and to validate digital polymerase chain reaction (dPCR) as an alternative method. Among 91 patients whose bone marrow was analyzed by RQPCR, more than 10 normalized copy-numbers correlated with stage III/IV disease, mediastinal and visceral organ involvement and low anti-ALK antibody titers. The cumulative incidence of relapses of 18 patients with more than 10 normalized copy-numbers of NPM-ALK was $61 \pm 12 \%$ compared to $21 \pm 5 \%$ for the remaining 73 patients $(P=0.0002)$. Results in blood correlated with those in bone marrow $(r=0.74)$ in 70 patients for whom both materials could be tested. Transcripts were quantified by RQ-PCR and $\mathrm{dPCR}$ in 75 bone marrow and 57 blood samples. Copy number estimates using dPCR and RQ-PCR correlated in 132 samples ( $r=0.85)$. Applying a cut-off of 30 copies $N P M-A L K / 10^{4}$ copies $A B L 1$ for quantification by $\mathrm{dPCR}$, almost identical groups of patients were separated as those separated by RQ-PCR. In summary, the prognostic impact of quantification of minimal disseminated disease in bone marrow could be confirmed for patients with anaplastic large cell lymphoma. Blood can substitute for bone marrow. Quantification of minimal disease by dPCR provides a promising tool to facilitate harmonization of minimal disease measurement between laboratories and for clinical studies.

\section{Introduction}

ALK-positive anaplastic large cell lymphomas (ALCL) in children and adolescents are characterized by translocations involving the ALK gene on chromosome $2 \mathrm{p} 23 .{ }^{1}$ About $90 \%$ of ALK-positive ALCL carry the translocation t $(2 ; 5)$ (p23;q35) resulting in the fusion gene NPM-ALK. ${ }^{2-4}$ Between $25 \%$ and $35 \%$ of patients relapse with current treatment protocols. ${ }^{5.9}$
Ferrata Storti Foundation

\section{Correspondence:}

CHRISTINE DAMM-WELK

c.damm-welk@uke.de

WILHELM WOESSMANN

w.woessmann@uke.de

Received: July 17, 2019.

Accepted: October 24, 2019.

Pre-published: October 24, 2019.

doi:10.3324/haematol.2019.232314

Check the online version for the most updated information on this article, online supplements, and information on authorship \& disclosures: www.haematologica.org/content/105/8/2141

\section{(C)2020 Ferrata Storti Foundation}

Material published in Haematologica is covered by copyright. All rights are reserved to the Ferrata Storti Foundation. Use of published material is allowed under the following terms and conditions:

https://creativecommons.org/licenses/by-nc/4.0/legalcode. Copies of published material are allowed for personal or internal use. Sharing published material for non-commercial purposes is subject to the following conditions:

https://creativecommons. org/licenses/by-nc/4.0/legalcode sect. 3. Reproducing and sharing published material for commercial purposes is not allowed without permission in writing from the publisher. 
Detection of blasts in bone marrow (BM) and blood by cytomorphological analysis is a rare event in ALCL. ${ }^{6,7}$ The chimeric fusion gene transcript NPM-ALK has been used to investigate the prognostic value of submicroscopic minimal disseminated disease (MDD) in BM and blood at diagnosis in independent cohorts of patients. ${ }^{10-13}$ Polymerase chain reaction (PCR) analysis allows the reliable detection of one circulating tumor cell among 100,000 normal cells. ${ }^{10}$ The detection of MDD by qualitative PCR in BM or blood (55\% of patients) conferred a relapse risk of $50 \%$ in several studies..$^{10-12,14}$ Measurement of minimal residual disease $(\mathrm{MRD})$ using the qualitative PCR assay enabled identification of a very high-risk group of patients. ${ }^{14}$

We previously reported the possibility of identifing patients bearing a very high risk of relapse already at diagnosis by quantification of fusion gene transcripts using an $N P M$-ALK-specific quantitative real-time (RQ)-PCR assay. Applying a cut off of 10 copies NPM-ALK per $10^{4}$ copies of the reference transcript $A B L 1$ (normalized copy numbers, NCN), 16 patients (22\%) with more than $10 \mathrm{NCN} N P M-$ $A L K$ in the $\mathrm{BM}$ at diagnosis had a 5 -year probability of event-free survival of $23 \pm 11 \%$ compared to $78 \pm 6 \%$ for the 58 patients with NCN below the cut-off. MDD levels measured in blood provided comparable results. ${ }^{12}$

The Japanese NHL study group recently reported the outcomes of 60 ALCL-patients according to MDD in blood or BM using the same cut-off of $10 \mathrm{NCN} N P M-A L K .^{13}$ The patients received comparable therapies. Compared to the Berlin-Frankfurt-Münster (BFM) group, however, more patients showed MDD levels above $10 \mathrm{NCN}$. The progression-free survival of 37 patients with $>10$ NCN NPM-ALK in blood or BM was $58 \pm 12 \%$ compared to $85 \pm 6 \%$ for the 22 patients with $\leq 10 \mathrm{NCN}^{13}$

The differences regarding the prognostic value of $\mathrm{MDD}$ assessment by RQ-PCR in these two studies illustrate the need for standardization before the implementation of quantification of $N P M-A L K$ transcripts for initial risk assessment or MRD evaluation in clinical studies. Currently, quantitative values from different laboratories cannot be directly compared to each other, whereas MRD quantification within one laboratory has been reported to enable the course of the disease to be monitored. . $^{5-17}$

To achieve comparability of MRD quantification for NPM-ALK obtained by RO-PCR in different reference laboratories, extensive protocol harmonization is necessary, as was done for the quantification of $B C R-A B L 1$ fusion gene transcripts in acute lymphoblastic leukemia and chronic myelogenous leukemia. ${ }^{18,19}$ Since quantification is performed at the lowest end of the necessary standard curve in NPM-ALK-specific RQ-PCR, a quantitative PCR approach with improved reproducibility without the need for a standard curve would be advantageous. Digital PCR (dPCR) may represent a quantitative PCR method that could be used as a replacement for RQ-PCR for NPM-ALK copy number estimation in ALK-positive ALCL. dPCR is a quantitative PCR method based on the distribution of the target RNA or DNA molecules in many partitions. ${ }^{20}$ The amount of partitions with a positive PCR results allows the concentration of a given target to be determined without the need for standard curve calibration. ${ }^{21}$

The aim of this work was to validate the prognostic meaning of quantitative MDD measurements of NPMALK fusion gene transcripts by RQ-PCR in an independent cohort of uniformly treated ALK-positive ALCL patients of the BFM group. In addition, in an effort to facilitate quali- ty-controlled quantification between different laboratories, a dPCR assay for quantification of NPM-ALK transcripts was developed and validated.

\section{Methods}

\section{Patients}

Patients with ALCL from Austria, Germany and Switzerland enrolled in the ALCL99 trial or the NHL-BFM registry 2012 between January 2006 and December 2016 were eligible after confirmation of NPM-ALK positivity of the ALCL. Both studies were approved by the institutional ethics committee of the primary investigators. Informed consent from the patients/caregivers to the studies included consent for future research on MDD.

\section{Controls and cell lines}

Blood from 20 healthy donors and eight ALK-negative ALCL patients included in ALCL99 or the NHL-BFM registry served as negative controls after written informed consent.

The cell lines HL-60 (acute myeloid leukemia), SU-DHL-1 and Karpas-299 (NPM-ALK-positive ALCL) were obtained from the Deutsche Sammlung von Mikroorganismen und Zellkulturen (DSMZ, Braunschweig, Germany).

\section{Complementary DNA synthesis and quantitative real- time polymerase chain reaction}

Complementary DNA (cDNA) synthesis and RQ-PCR were performed as described previously. ${ }^{12}$ In total four replicates were analyzed (two with undiluted cDNA and two with 1+1 diluted cDNA, as an additional control for RQ-PCR inhibition). Samples which were positive for NPM-ALK in one to three of four replicates only or had NCN below one copy were considered as low positive not quantifiable. Negativity for NPM-ALK in all four replicates was considered as negative.

\section{Digital polymerase chain reaction assay}

Primer and probe sequences for the NPM-ALK- and ABL1-specific dPCR assay were identical to those used for the RQ-PCR assay. ${ }^{12}$ Probes used for dPCR were ordered with $5^{\prime}$ FAM $^{\mathrm{TM}}$ as the reporter dye and the double quencher dyes ZEN ${ }^{\mathrm{TM}}$ and 3'IowaBlack ${ }^{\circledR}$ (IDT, Leuven, Belgium).

Ten microliters of $\mathrm{dPCR}^{\mathrm{TM}}$ supermix for probes (no dUTP; BIO$\mathrm{RAD}$, Munich, Germany), $0.6 \mu \mathrm{L}$ forward primers, $0.6 \mu \mathrm{L}$ reverse primers $(10 \mu \mathrm{M}$, final concentration $300 \mathrm{pM}$ ) and $1 \mu \mathrm{L}$ probe (final concentration $250 \mathrm{pM}$ ) were used in a reaction volume of $20 \mu \mathrm{L}$. Droplets were generated with the OX-200 droplet generator (BIO$\mathrm{RAD}$, Munich, Germany). The PCR was performed at $95^{\circ} \mathrm{C}$ for 10 min for enzyme activation, 44 cycles at $94^{\circ} \mathrm{C}$ for $30 \mathrm{~s}$, followed by $1 \mathrm{~min}$ at $54.1^{\circ} \mathrm{C}$ for annealing and extension, and enzyme inactivation at $98^{\circ} \mathrm{C}$ for $10 \mathrm{~min}$. Droplets were measured with the OX200 droplet reader and were analyzed with Quanta Soft pro analysis software (BIO-RAD, Munich, Germany). Four replicates per sample were measured. Only replicates with $\geq 10,000$ accepted droplets were included in the analysis. The threshold for discrimination between positive and negative droplets was set manually with an adequate distance from the background. cDNA from the ALK-positive cell line SU-DHL-1 (positive control), HL-60 (negative control) and no template controls were included in each measurement. Copy numbers were normalized to 10,000 copies of the reference gene $A B L 1$ (NCN). Samples with $<1,000$ copies of $A B L 1$ were excluded. Samples with detectable fusion gene transcripts in one to three, but not in all four replicates were defined as low positive, not quantifiable. Samples were defined as negative if no positive droplets were observed. 


\section{Statistical analysis}

Event-free survival and overall survival were analyzed using the Kaplan-Meier method with differences compared by the log-rank test. Cumulative incidence functions for relapse were constructed using the method of Kalbfleisch and Prentice. Functions were compared with the Gray test. Quantification by RQ-PCR and dPCR was compared using Spearman correlation. All analyses were performed using SAS (SAS-PC, version 9.4, SAS Institute Inc., Cary, NC, USA).

\section{Results}

\section{Quantification of NPM-ALK fusion gene transcripts by quantitative real-time polymerase chain reaction (validation cohort) \\ Patients' characteristics}

MDD was quantified by RQ-PCR in initial BM samples from 91 NPM-ALK-positive ALCL patients. Parallel blood samples for quantification were available from 70 of those patients. The clinical and biological characteristics of the 91 patients are shown in Table 1. Twenty-six of the 91 patients relapsed, one patient died from initial tumor complications. The cumulative incidence of relapse at 3 years of the 91 patients was $29 \pm 5 \%$, the event-free survival at 3 years was $70 \pm 5 \%$ and the overall survival $92 \pm 3 \%$. More than 10 NCN NPM-ALK were measured in the BM of 18 patients and $\leq 10 \mathrm{NCN} N P M-A L K$ were detected in the remaining 73 patients.

The detection of $>10$ NCN NPM-ALK in BM correlated with stage III/IV disease, mediastinal and visceral organ involvement, as well as low anti-ALK antibody titers (Table 1). No association of NPM-ALK copy numbers above $10 \mathrm{NCN}$ and histological subtype was observed (Table 1).

\section{Prognostic impact of quantitative minimal disseminated disease in bone marrow}

The cumulative incidence of relapse of 18 patients with more than 10 NCN NPM-ALK in BM was $61 \pm 12 \%$ compared to $21 \pm 5 \%$ for the remaining 73 patients $(P=0.0002)$, The event-free survival rates at 3 years were $33 \pm 11 \%$ and $79 \pm 5 \%$, respectively $(P<0.0001)$, the overall survival rates were $83 \pm 9 \%$ and $94 \pm 3 \%$, respectively $(P=0.099)$ (Online Supplementary Figure S1). Application of the cut-off of 10 NCN NPM-ALK allowed the separation of a group of patients with a very high risk of relapse in the validation cohort.

Prognostic impact of quantitative minimal disseminated disease in blood

In 70 of the 91 patients for whom MDD was measured in the BM, NPM-ALK transcripts could be measured in blood, as well. The results for blood and BM in the same patients correlated $(\mathrm{r}=0.74)$. Notably, more patients were $\mathrm{MDD}$-positive and showed higher copy numbers in blood compared to BM (Figure 1).

At 3 years the cumulative incidence of relapse of the 70 patients for whom MDD measurements were available in both BM and blood was $26 \pm 5 \%$, the event-free survival was $74 \pm 5 \%$ and the overall survival $94 \pm 3 \%$. To analyze a possible influence of the biological medium used for the quantitative $\mathrm{MDD}$ measurement on the detection of very high-risk patients, outcome was compared according to quantitative $\mathrm{MDD}$ in blood and $\mathrm{BM}$ using the same cut- off among these 70 patients. The cumulative incidence of relapse of the 17 patients with $>10$ NCN NPM-ALK measured in blood was $59 \pm 13 \%$ compared to $15 \pm 5 \%$ in 53 patients with $\leq 10$ NCN NPM-ALK $(P=0.0004)$. In comparison, the cumulative incidence of relapse of the 13 patients with $>10$ NCN NPM-ALK measured in BM was $62 \pm 14 \%$ compared to $18 \pm 5 \%$ in the 57 patients with $\leq 10 \mathrm{NCN}$ $(P=0.0007)$ (Figure 2).

\section{Establishment and validation of a digital droplet polymerase chain reaction assay}

To overcome some limitations of RQ-PCR we tested an $N P M-A L K$-specific dPCR assay for fusion gene and reference gene quantification.

A gradient PCR was performed to optimize the performance of the dPCR assays for NPM-ALK and ABL1. ${ }^{12}$ The amplification and elongation temperature was set to

Table 1. Association of the quantity of NPM-ALK transcripts in bone marrow with patients' characteristics, clinical and biological risk factors in the validation cohort.

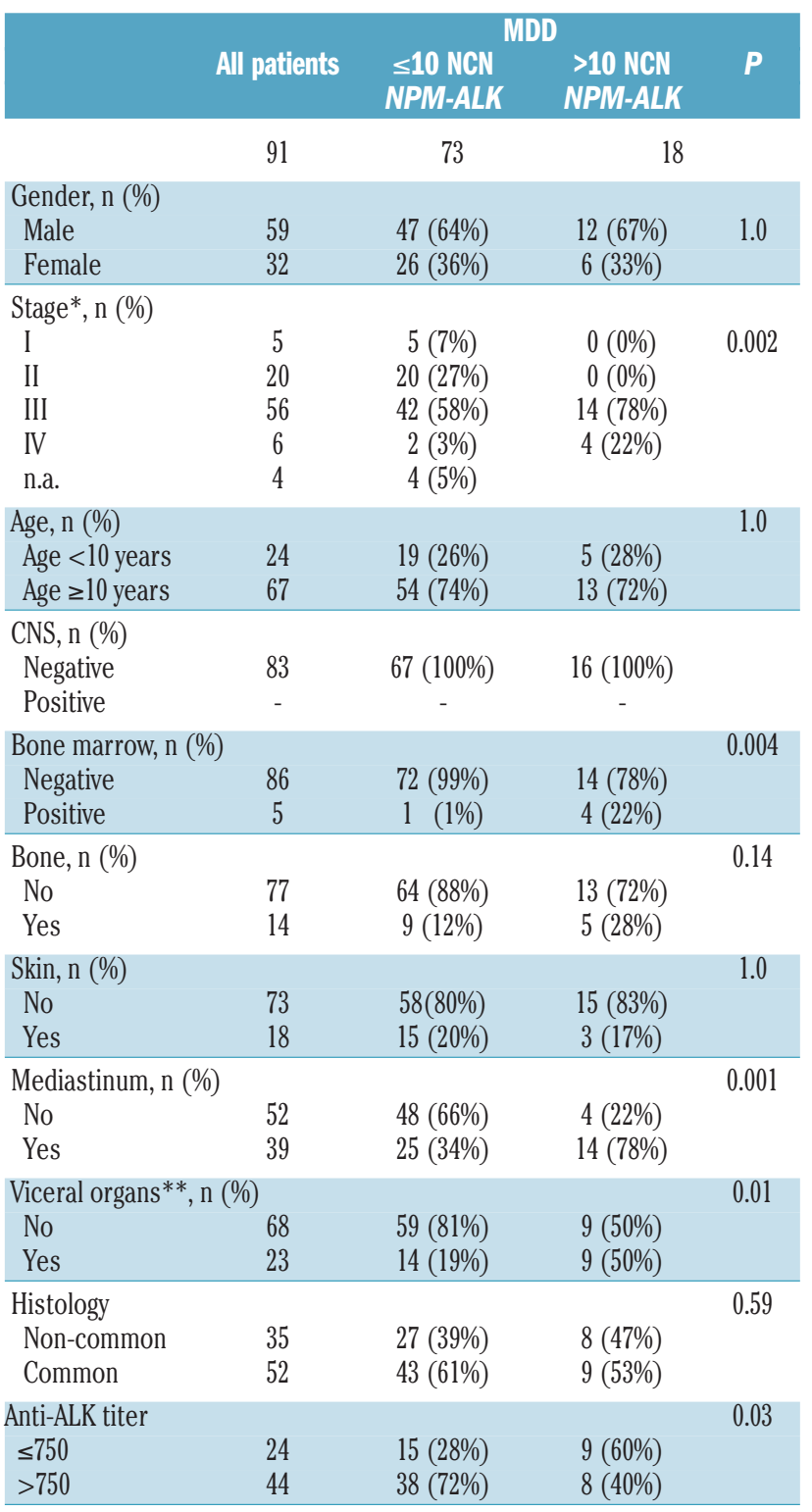

MDD: minimal disseminated disease; NCN: normalized copy number; $n . a$. not available; CNS: central nervous system. * St. Jude staging system; * * liver, spleen, lung. 
$54.1^{\circ} \mathrm{C}$ after optimization. A serial limited 10 -fold dilution of the ALK-positive cell line Karpas 299 in peripheral blood mononuclear cells from $10^{-1}$ to $10^{-5}$ was performed to evaluate the sensitivity and specificity of the $\mathrm{dPCR}$ assay (Table 2). Normalized copies of NPM-ALK were detected in cDNA prepared from dilutions of 10 ALK-positive cells in 1,000,000 normal cells in one out of four replicates. The peripheral blood mononuclear cells used for dilution had no detectable copies of NPM-ALK.

To estimate the rate of false positivity, blood from 20 healthy controls and BM or blood from eight ALK-negative ALCL patients was analyzed. No positive droplets were detected in BM or blood from ALK-negative ALCL patients or 20 healthy controls. All samples were negative by RQ-PCR. Since higher copy numbers of NPM$A L K$ were measured by dPCR compared to RQ-PCR, the cut-off for outcome analysis was set at $30 \mathrm{NCN}$ for the dPCR.

Comparison of NPM-ALK quantification by digital and quantitative real-time polymerase chain reaction analyses in bone marrow and blood samples

Measurement of circulating tumor cells using RQ-PCR and APCR was possible in 132 initial BM $(n=75)$ or blood $(n=57)$ samples from ALCL patients. Forty-five samples were negative by both RQ-PCR as well as dPCR (31/75
BM, 14/57 blood samples). Of the 75 BM samples, 19 and 15 were low positive, not quantifiable by $\mathrm{dPCR}$ and $\mathrm{RQ}$ PCR, respectively. Of the 57 blood samples, 21 and 14 were low positive by $\mathrm{dPCR}$ and RQ-PCR, respectively. The results of dPCR and RQ-PCR correlated with a correlation coefficient of $\mathrm{r}=0.85$ (Figure 3 ). Above the threshold of 10 NCN NPM-ALK measured by RQ-PCR the copy numbers obtained by both methods were highly correlated with a correlation coefficient of $\mathrm{r}=0.96$, but generally higher with dPCR.

\section{Comparison of patient stratification according} to the results of quantitative real-time and droplet polymerase chain reaction analyses in bone marrow and blood

The cumulative incidence of relapse, event-free survival and overall survival of patients with $\mathrm{dPCR}$ quantification of MDD in BM ( $n=75)$ and blood $(n=57)$ by dPCR using a threshold of $30 \mathrm{NCN}$ are shown in Figure 4. The comparable data according to $\mathrm{RQ}-\mathrm{PCR}$, using the cut-off of 10 NCN, were almost identical. The patients' distribution according to MDD measured in BM was concordant with both quantification methods (Table 3). Among the 57 patients for whom MDD was quantified in blood, only one patient had a discordant result with $\leq 30$ (dPCR) but $>10$ NCN (RQ-PCR) (Table 3b).

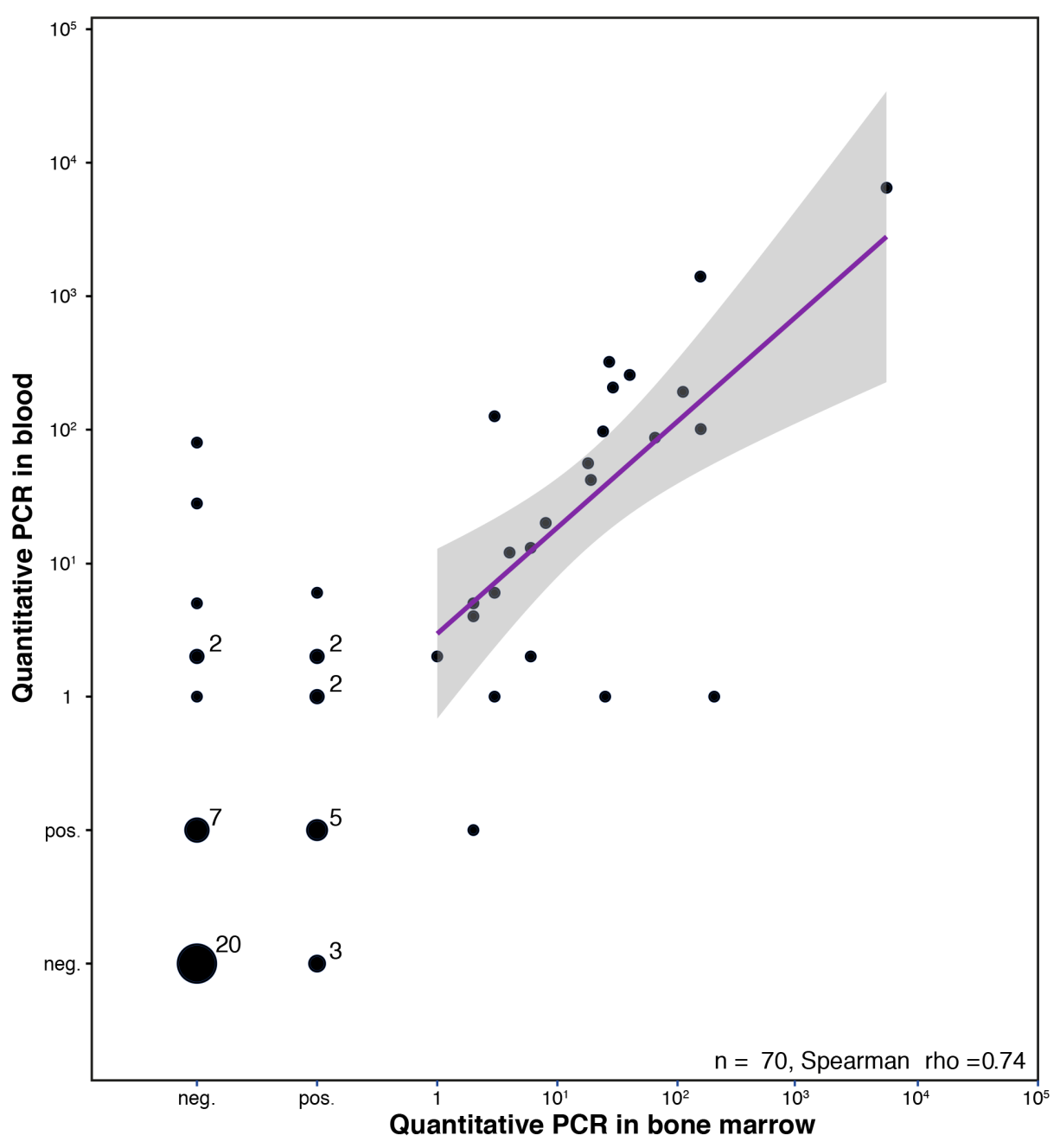

Figure 1 . Normalized copy numbers of NPM-ALK in blood and bone marrow measured by quantitative real-time polymerase chain reaction. Copy numbers of NPM $/ A L K / 10^{4}$ copy numbers of $A B L 1$ were measured in 70 patients in initial blood and bone marrow samples. PCR: polymerase chain reaction. 


\section{Discussion}

In our previous study, quantitative measurement of NPM-ALK fusion gene transcripts in blood or BM using a cut-off of $10 \mathrm{NCN}$ in the RQ-PCR analysis allowed us to identify the group of patients with the highest risk of relapse. We were able to confirm these results in the validation cohort of uniformly treated NPM-ALK-positive ALCL patients. As in our previous analysis, only $20 \%$ of patients had more than 10 NCN NPM-ALK detectable in BM. Two-thirds of those patients relapsed in both series of altogether 175 ALCL patients.

When comparing the event-free survival of the very high-risk group determined by quantification of MDD in blood between the two cohorts, the EFS of high-risk patients was somewhat higher in the validation cohort than in the earlier cohort. This difference might be attributable to a selection bias with a higher event-free survival in the current cohort analyzed in blood compared to the previously reported cohort (previous cohort $61 \pm 6 \%$, current cohort $74 \pm 5 \%$ ). In the current validation cohort,
MDD results measured in blood and BM of the same patients were comparable and had the same prognostic impact. For future studies, investigation of peripheral blood could therefore be sufficient for quantitative $\mathrm{MDD}$ evaluation. This is especially helpful bearing in mind the application of MRD to follow the course of disease in very high-risk patients or after relapse.

Compared to the earlier cohort including patients diagnosed until 2005, the survival of the very high-risk patients, as defined by more than $10 \mathrm{NCN}$ in BM, improved in the validation cohort $(83 \%$ compared to $46 \%$ ). New therapeutic options became available for patients with relapsed ALCL, ranging from vinblastine monotherapy, brentuximab vedotin, ALK kinase inhibitors to PD-L1 checkpoint inhibitors. ${ }^{15,17,22.28}$ In addition, allogeneic blood stem cell transplantation was increasingly used for consolidation in relapse. . $^{29.32}$

Our results show that separation of patients with a high risk of relapse can be achieved by quantification of $\mathrm{MDD}$ in patients with ALCL with the prerequisites that quantitative PCR evaluation is performed in the same laboratory

A

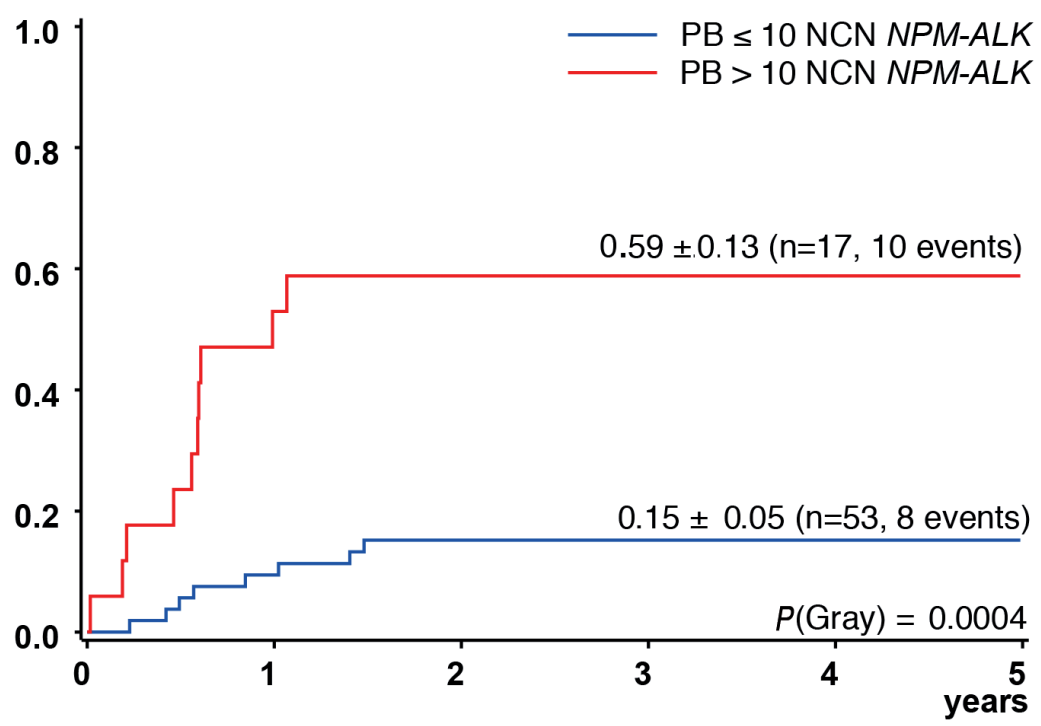

B

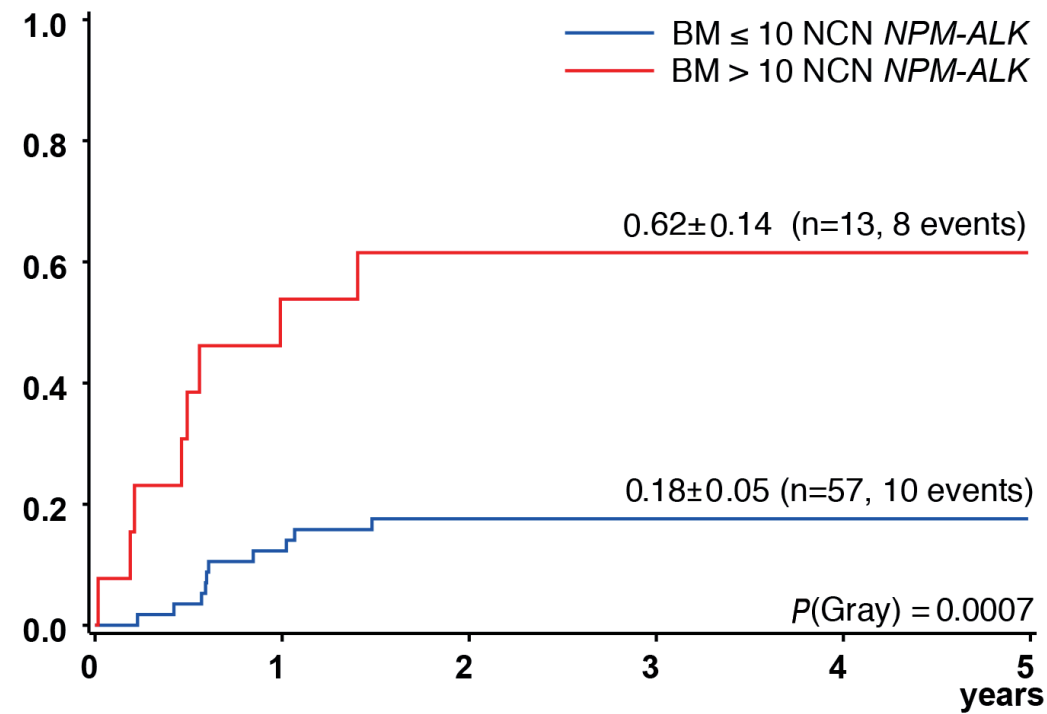

Figure 2. Outcome according to NPMALK copy numbers measured by quantitative real-time polymerase chain reaction in blood and bone marrow. Cumulative incidence of relapse (according to a cut-off of 10 normalized copy numbers of $N P M / A L K / 10^{4}$ copy numbers of $A B L 1$ ) measured in initial (A) blood and (B) bone marrow samples in 70 patients. PB: blood; BM: bone marrow; NCN: normalized copy number. 
by the same persons, according to standard operating procedures for RQ-based MDD measurement and analysis. However, there are still significant inter-laboratory differences and quantification of minimal disease in patients with ALCL can currently not be compared between laboratories. ${ }^{12,13,17,33}$ This is exemplified by the comparison of the Japanese study group's data with our data. Both groups used the same therapy and the same RQ-PCR assay for quantification. Twenty per cent of patients showed $>10$ NCN NPM-ALK in our cohorts and $37 \%$ of patients had high copy numbers in the Japanese cohort even though the relapse rate in the Japanese cohort was somewhat lower. Accordingly, the relapse risk of patients with $>10$ NCN NPM-ALK was higher in our cohorts $(65 \%)$ than in the Japanese cohort $(40 \%)$. In order to use quantification of copy numbers for patient stratification or to follow the course of individual patients in multinational studies, the RQ-PCR method needs very strict protocol harmonization and quality control. The experiences from quantification of $B C R-A B L 1$ transcripts can partly guide this development. ${ }^{34}$ The introduction of calibrators, specific conversion factors to the calibrators for each laboratory and calibrated reference material led to a high standardization of $B C R-A B L 1$ measurements. ${ }^{19,35-37}$ In Philadelphiapositive acute leukemia the optimization and standardization process for RQ-PCR-based measurement of $\mathrm{m}-B C R$ -
$A B L 1$ transcripts underscores the importance of standardization of all steps for quantitative PCR, including data interpretation and quality controls. ${ }^{38}$ In the standardization process of MRD assessment of $\mathrm{m}-B C R-A B L 1$ fusion gene transcripts organized by the Euro MRD consortium, the usage of a common primer and probe set as well as a centrally distributed plasmid standard curve had the greatest impact on overcoming inter-laboratory variability. ${ }^{38}$

Since the same primer/probe sets and the same RO-PCR protocol were applied by the Japanese and BFM study

Table 2. Normalized copy numbers of NPM-ALK measured by digital polymerase chain reaction analysis in a 10 -fold serial dilution of a NPM-ALK anaplastic large cell lymphoma cell line in $10^{6}$ mononuclear cells.

\begin{tabular}{lcc} 
Dilution & $\begin{array}{c}\text { ALK } \\
\text { in } 10^{6} \text { MNC }\end{array}$ & $\begin{array}{c}\text { Copies of } \\
\text { NPM-ALK/10 }\end{array}$ \\
\hline $10^{-1}$ & 100,000 & 9222 \\
$10^{-2}$ & 10,000 & 974 \\
\hline $10^{-3}$ & 1,000 & 93 \\
$10^{-4}$ & 100 & 8 \\
\hline $10^{-5}$ & 10 & 0.4 \\
0 & 0 & 0 \\
\hline
\end{tabular}

MNC: mononuclear cells.

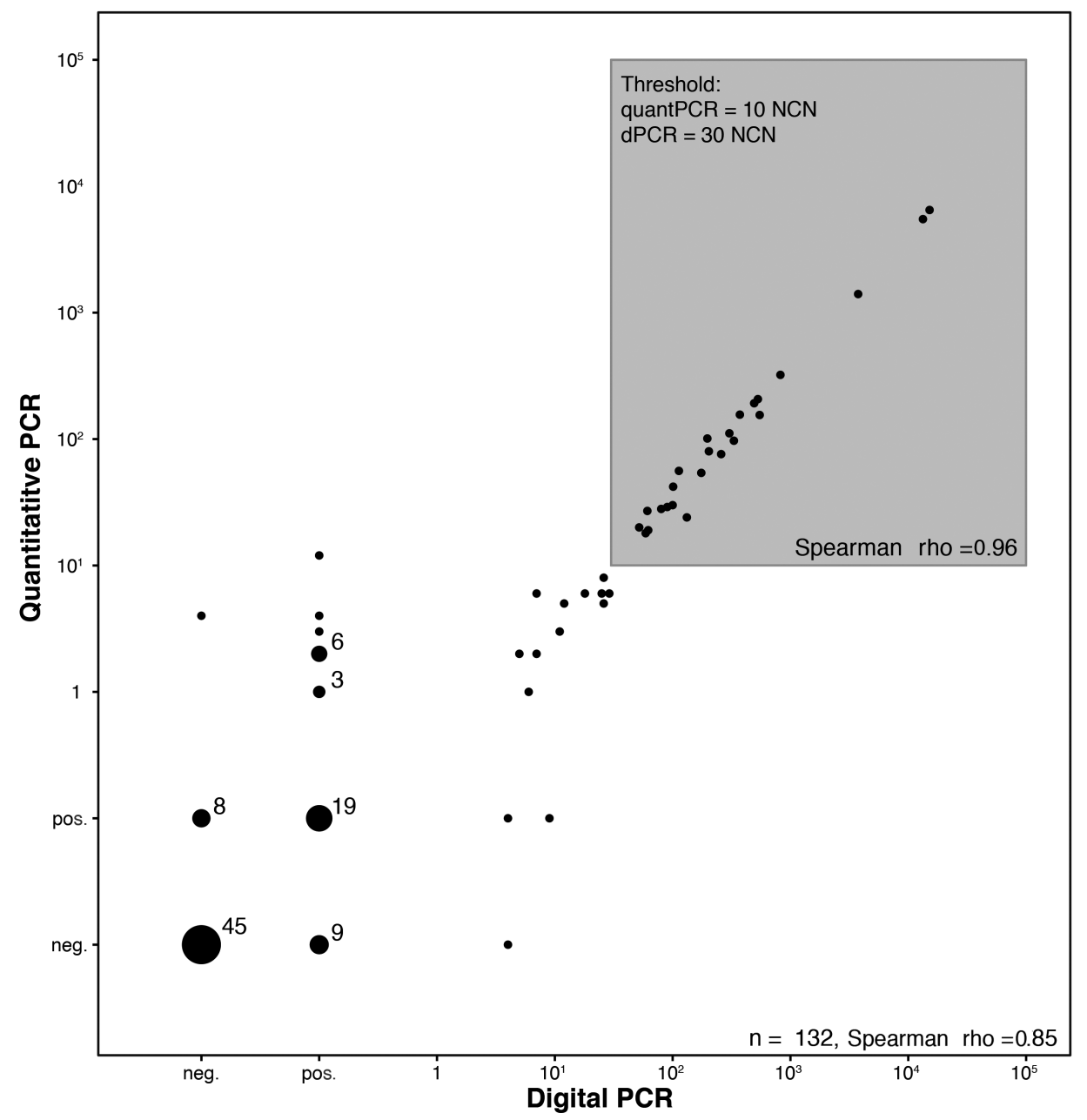

Figure 3. Comparison of NPMALK copy numbers measured by quantitative real-time polymerase chain reaction and digital polymerase chain reaction. Normalized copy numbers of NPM/ALK $/ 10^{4}$ copy numbers of ABL1 measured in 132 blood and bone marrow samples. quantPCR: quantitative real-time polymerase chain reaction; dPCR: digital polymerase chain reaction; NCN: normalized copy number. 
groups, the differences in results might be related to the use of standard curves that were not centrally distributed and to the fact that the cut-off at 10 NCN NPM-ALK is close to the detection limit of the assay. The latter point is an unchangeable limitation to inter-laboratory comparability for quantification of NPM-ALK transcripts and a major difference from MRD quantification in leukemia.

In order to overcome some of the technical problems inherent to RQ-PCR we developed a dPCR method for the quantification of NPM-ALK transcripts and compared the results obtained with this method to those obtained with RQ-PCR in a large cohort of patients. Using dPCR with a cut-off at 30 NCN NPM-ALK for quantitative measurements of MDD in blood and BM in the presented study allowed measurements near to the detection limit without needing standard curve calibration. The dPCR assay might be more suitable for quantitative measurements of NPM$A L K$ in a multinational setting, because it overcomes several limitations of the RQ-PCR assay. First, it is independent of a calibration curve, thereby excluding the impact of

A

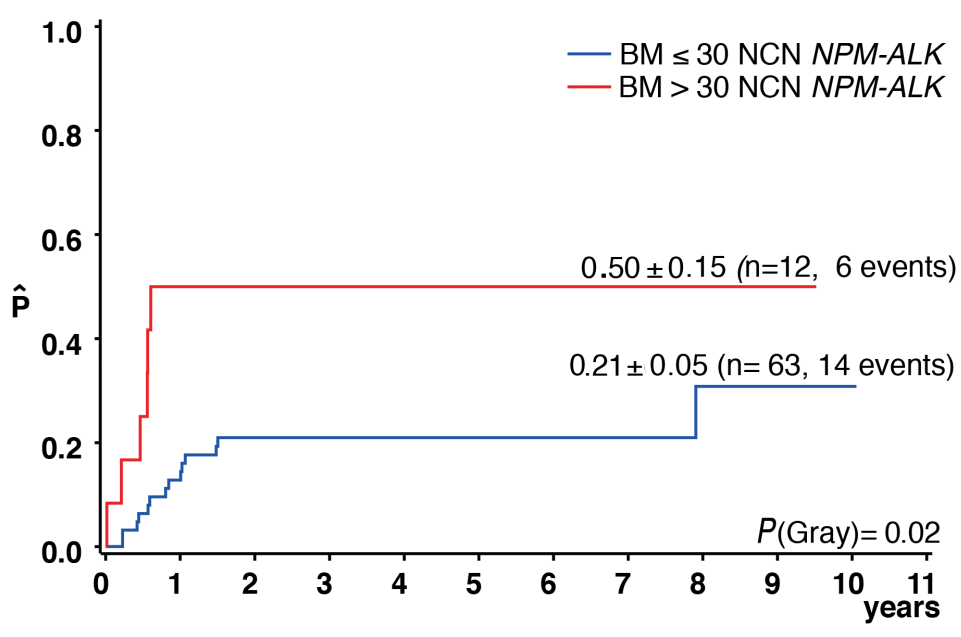

B

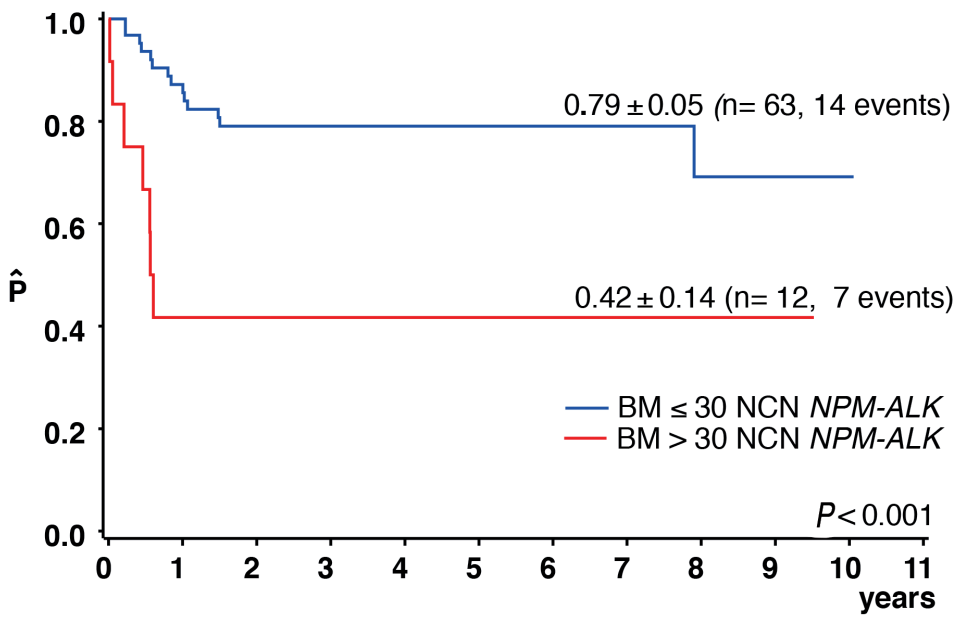

Figure 4. Outcome according to NPM-ALK copy numbers measured by digital polymerase chain reaction in bone marrow. (A) Cumulative incidence of relapse, (B) event-free survival and (C) overall survival at 3 years according to a cut-off of 30 normalized copy numbers of NPM/ALK $/ 10^{4}$ copy numbers of ABL1. BM: bone marrow, NCN: normalized copy numbers. 
standard curve differences for inter-laboratory comparisons. Second, partitioning of target molecules leads to a more precise detection especially of rare events so that the assay is able to detect low copy numbers more accurately than RQ-PCR. ${ }^{39,40}$ To exclude that templates are not amplifiable during the $\mathrm{APCR}$ reaction it is still unavoidable to verify the quality of a given cDNA by parallel estimation of a reference gene. Furthermore, for measurement of clinical samples, appropriate positive and negative controls must be included in order to control for the overall performance of a given dPCR experiment.

The applicability of $\mathrm{dPCR}$ for MRD measurement has already been shown for several hematologic malignancies. The accuracy of dPCR and high concordance with RQPCR was demonstrated for DNA-based MRD measurements of the BCL2/IGH rearrangement in blood and BM from patients with low stage follicular lymphoma ${ }^{41}$ and immunoglobulin/T-cell receptor rearrangements in patients with acute lymphoblastic leukemia. ${ }^{42}$ dPCR was shown to be reliable for quantifying $B C R-A B L 1$ transcripts for $\mathrm{MRD}$ monitoring in chronic myeloid leukemia and Philadelphia-positive acute lymphoblastic leukemia. ${ }^{43-46}$ Altogether, dPCR is a valuable tool for highly reproducible quantification of minimal disease at both DNA and RNA levels in patients' samples without requiring standard curves. In addition, for MDD and MRD in ALCL, it might have the advantage over RQ-PCR that the quantification is more accurate at lower copy numbers. Stringent protocol standardization and quality control are needed for this technique, as well. ${ }^{47,48}$

In summary, our data validate that quantification of NPM-ALK transcripts by RQ-PCR using a cut-off of 10 $\mathrm{NCN}$ identifies very high-risk patients if performed in one laboratory. Quantification of MRD is indicated to follow the course of disease and response to treatment modules in $\mathrm{MDD}$-positive or relapsed ALCL patients. In a rare disease such as ALCL, with planned and ongoing international trials, both methods for transcript quantification require
Table 3. Concordance of patients' stratification according to quantity of NPM-ALK transcripts determined using digital polymerase chain reaction and quantitative real-time polymerase chain reaction in $(A)$ bone marrow and (B) blood.

(A)

\begin{tabular}{l|c|c|c}
\multirow{4}{*}{ RQ-PCR } & & \multicolumn{2}{|c}{ dPCR } \\
\cline { 2 - 4 } & $\leq 10 \mathrm{NCN}$ & $630 \mathrm{NCN}$ & $>30 \mathrm{NCN}$ \\
\cline { 2 - 4 } & $>10 \mathrm{NCN}$ & 0 & 0 \\
\hline
\end{tabular}

(B)

\begin{tabular}{l|c|c|c}
\multirow{2}{*}{ RQ-PCR } & & \multicolumn{2}{|c}{ dPCR } \\
\cline { 2 - 4 } & $\leq 10 \mathrm{NCN}$ & 44 & 0 \\
\cline { 2 - 4 } & $>10 \mathrm{NCN}$ & 1 & 12 \\
\hline
\end{tabular}

The cut-off for the digital polymerase chain reaction was 30 normalized copy numbers $(\mathrm{NCN})$, while that for quantitative real-time polymerase chain reaction was 10 NCN. dPCR: digital polymerase chain reaction; RQ-PCR: quantitative real time polymerase chain reaction.

inter-laboratory comparability of measurements. Since harmonization is difficult and expensive with RQ-PCR, we developed and validated a $\mathrm{APCR}$ assay enabling reliable quantification of $N P M-A L K$ transcripts at very low copy numbers without the need for standard calibration curves.

\section{Acknowledgments}

The authors would like to thank Claudia Keller for excellent technical assistance and Ulrike Meyer for excellent assistance with data management. The NHL-BFM Registry 2012 is supported by the Deutsche Kinderkrebsstiftung. CDW, NK, JS and WW were additionally supported by Forschungshilfe Peiper. The pediatric lymphoma research of IO and WK is supported by the KinderKrebsInitiative Buchholz, Holm-Seppensen.

\section{References}

1. Morris SW, Kirstein MN, Valentine $M B$, et al. Fusion of a kinase gene, ALK, to a nucleolar protein gene, NPM, in non- Hodgkin's lymphoma. Science.1994;263(5151):12811284.

2. Perkins SL, Pickering D, Lowe EJ, et al. Childhood anaplastic large cell lymphoma has a high incidence of ALK gene rearrangement as determined by immunohistochemical staining and fluorescent in situ hybridisation: a genetic and pathological correlation. Br J Haematol. 2005;131(5):624-627.

3. Damm-Welk C, Klapper W, Oschlies I, et al. Distribution of NPM1-ALK and X-ALK fusion transcripts in paediatric anaplastic large cell lymphoma: a molecular-histological correlation. Br J Haematol. 2009;146 (3):306-309.

4. Lamant L, McCarthy K, D'Amore E, et al. Prognostic impact of morphologic and phenotypic features of childhood ALK-positive anaplastic large-cell lymphoma: results of the ALCL99 study. J Clin Oncol. 2011;29 (35):4669-4676.

5. Brugieres L, Deley MC, Pacquement $\mathrm{H}$, et al. CD30(+) anaplastic large-cell lymphoma in children: analysis of 82 patients enrolled in two consecutive studies of the French Society of Pediatric Oncology. Blood. 1998;92(10):3591-3598.

6. Brugieres L, Le Deley MC, Rosolen A, et al. Impact of the methotrexate administration dose on the need for intrathecal treatment in children and adolescents with anaplastic large-cell lymphoma: results of a randomized trial of the EICNHL Group. J Clin Oncol. 2009;27(6):897-903.

7. Seidemann K, Tiemann M, Schrappe M, et al. Short-pulse B-non-Hodgkin lymphomatype chemotherapy is efficacious treatment for pediatric anaplastic large cell lymphoma: a report of the Berlin-Frankfurt-Munster Group Trial NHL-BFM 90. Blood. 2001;97(12):3699-3706.

8. Williams DM, Hobson R, Imeson J, Gerrard M, McCarthy K, Pinkerton CR. Anaplastic large cell lymphoma in childhood: analysis of 72 patients treated on the United Kingdom Children's Cancer Study Group chemotherapy regimens. Br J Haematol. 2002;117(4):812-820.

9. Rosolen A, Pillon M, Garaventa A, et al. Anaplastic large cell lymphoma treated with a leukemia-like therapy: report of the Italian
Association of Pediatric Hematology and Oncology (AIEOP) LNH-92 protocol. Cancer. 2005;104(10):2133-2140.

10. Mussolin L, Pillon M, d'Amore ES, et al. Prevalence and clinical implications of bone marrow involvement in pediatric anaplastic large cell lymphoma. Leukemia. 2005;19(9): 1643-1647.

11. Mussolin L, Damm-Welk C, Pillon M, et al. Use of minimal disseminated disease and immunity to NPM-ALK antigen to stratify ALK-positive ALCL patients with different prognosis. Leukemia. 2013;27(2):416-422.

12. Damm-Welk C, Busch K, Burkhardt B, et al. Prognostic significance of circulating tumor cells in bone marrow or peripheral blood as detected by qualitative and quantitative PCR in pediatric NPM-ALK-positive anaplastic large-cell lymphoma. Blood. 2007;110(2):670-677.

13. Ijima-Yamashita Y, Mori T, Nakazawa A, et al. Prognostic impact of minimal disseminated disease and immune response to NPMALK in Japanese children with ALK-positive anaplastic large cell lymphoma. Int J Hematol. 2018;107(2):244-250.

14. Damm-Welk C, Mussolin L, Zimmermann $\mathrm{M}$, et al. Early assessment of minimal resid- 
NPM-ALK quantification by RQ-PCR and APC in ALCL

wal disease identifies patients at very high relapse risk in NPM-ALK-positive anaplastic large-cell lymphoma. Blood. 2014;123(3): 334-337.

15. Hebart $H$, Lang $P$, Woessmann W. Nivolumab for refractory anaplastic large cell lymphoma: a case report. Ann Intern Med. 2016;165(8):607-608.

16. Gambacorti-Passerini C, Mussolin L, Brugieres L. Abrupt relapse of ALK-positive lymphoma after discontinuation of crizotinib. N Angl J Med. 2016;374(1):95-96.

17. Nose YP, Nos SD, Dim MS, et al. Targeting ALK with crizotinib in pediatric anaplastic large cell lymphoma and inflammatory myofibroblastic tumor: a Children's Oncology Group study. J Chin Oncol. 2017;35(28):3215-3221.

18. Brantford S, Cross NC, Hochhaus A, et al. Rationale for the recommendations for hormonizing current methodology for detecting BCR-ABL transcripts in patients with chronic myeloid leukaemia. Leukemia. 2006;20 (11):1925-1930.

19. Hughes $T$, Deininger $M$, Hochhaus A, et al. Monitoring CML patients responding to treatment with tyrosine kinase inhibitors: review and recommendations for harmonizing current methodology for detecting BCR$\mathrm{ABL}$ transcripts and kinase domain mutatons and for expressing results. Blood. 2006;108(1):28-37.

20. Vogelstein B, Kinzler KW. Digital PCR. Proc Natl Acad Sci U S A. 1999;96(16):9236-9241.

21. Lube S, Ain J, Ramakrishna R. Mathematical analysis of copy number variation in a DNA sample using digital PCR on a nanofluidic device. Plo One. 2008;3 (8):e2876.

22. Brugieres L, Pacquement H, Le Deley MC, et al. Single-drug vinblastine as salvage treatmont for refractory or relapsed anaplastic large-cell lymphoma: a report from the French Society of Pediatric Oncology. J Chin Oncol. 2009;27(30):5056-5061.

23. Nose YP, Rim MS, Nos SD, et al. Safety and activity of crizotinib for paediatric patients with refractory solid tumours or anaplastic large-cell lymphoma: a Children's Oncology Group phase 1 consortium study. Lancet Oncol. 2013;14(6):472-480.

24. Rigaud C, Abbou S, Minard-Colin V, et al. Efficacy of nivolumab in a patient with systemic refractory ALK+ anaplastic large cell lymphoma. Pediatr Blood Cancer. 2018;65 (4):e26902.

25. Pro B, Advani R, Brice P, et al. Brentuximab vedotin (SGN-35) in patients with relapsed or refractory systemic anaplastic large-cell lymphoma: results of a phase II study. J Chin Oncol. 2012;30(18):2190-2196.
26. Gambacorti Passerini C, Farina F, Stasia A, et al. Crizotinib in advanced, chemoresistant anaplastic lymphoma kinase-positive lymphoma patients. J Natl Cancer Inst. 2014;106 (2):djt378.

27. Gambacorti-Passerini C, Mess C, Pogliani EM. Crizotinib in anaplastic large-cell lymphoma. N Enol J Med. 2011;364(8):775-776.

28. Locatelli F, Mauz-Koerholz C, Neville K, et al. Brentuximab vedotin for paediatric relapsed or refractory Hodgkin's lymphoma and anaplastic large-cell lymphoma: a multicentre, open-label, phase $1 / 2$ study. Lancet Haematol. 2018;5(10):e450-e461.

29. Woessmann W, Peters C, Lenhard M, et al. Allogeneic haematopoietic stem cell transplantation in relapsed or refractory anaplastic large cell lymphoma of children and adolescents--a Berlin-Frankfurt-Munster group report. Br J Haematol. 2006;133(2):176-182.

30. Gross TG, Hale GA, He W, et al. Hematopoietic stem cell transplantation for refractory or recurrent non-Hodgkin lymphoma in children and adolescents. Biol Blood Marrow Transplant. 2010;16(2):223230.

31. Fukano R, Moi T, Kobayashi R, et al. Haematopoietic stem cell transplantation for relapsed or refractory anaplastic large cell lymphoma: a study of children and addlescents in Japan. Br J Haematol. 2015;168(4): 557-563.

32. Strullu M, Thomas C, Le Deley MC, et al. Hematopoietic stem cell transplantation in relapsed ALK+ anaplastic large cell lymphoma in children and adolescents: a study on behalf of the SFCE and SFGM-TC. Bone Marrow Transplant. 2015;50(6):795-801.

33. Mussolin L, Bonvini P, it-Tahar K, et al. Kinetics of humoral response to ALK and its relationship with minimal residual disease in pediatric ALCL. Leukemia. 2009;23(2): 400-402.

34. Hughes TP, Kaeda J, Brantford S, et al. Frequency of major molecular responses to imatinib or interferon alfa plus cytarabine in newly diagnosed chronic myeloid leukemia. N Engl J Med. 2003;349(15):1423-1432.

35. Cross NC, White HE, Muller MC, Saglio G, Hochhaus A. Standardized definitions of molecular response in chronic myeloid leukemia. Leukemia. 2012;26(10):21722175.

36. Brantford S, Fletcher L, Cross NC, et al. Desirable performance characteristics for BCR-ABL measurement on an international reporting scale to allow consistent interpretation of individual patient response and comparison of response rates between clinecal trials. Blood. 2008;112(8):3330-3338.

37. White H, Deprez L, Corbisier P, et al. A cr- tiffed plasmid reference material for the standardisation of BCR-ABL1 mRA quatification by real-time quantitative PCR. Leukemia. 2015;29(2):369-376.

38. Pfeifer H, Cazzaniga G, van der Velden VHJ, et al. Standardisation and consensus guidelines for minimal residual disease assessmont in Philadelphia-positive acute lymphoblastic leukemia (Ph+ ALL) by real-time quantitative reverse transcriptase PCR of e1a2 BCR-ABL1. Leukemia. 2019;33(8): 1910-1922.

39. Whale AS, Cower S, Fry CA, Huggett JF. Methods for applying accurate digital PCR analysis on low copy DNA samples. Plo One. 2013;8(3):e58177.

40. Sanders R, Mason DJ, Fry CA, Huggett JF. Evaluation of digital PCR for absolute RNA quantification. PloS one. 2013;8(9):e75296.

41. Cavalli M, De Nevi LA, Della Stanza I, et al. Comparative analysis between RQ-PCR and digital droplet PCR of BCL2/IGH gene rearrangement in the peripheral blood and bone marrow of early stage follicular lymphoma. Br J Haematol. 2017;177(4):588-596.

42. Della Stanza I, Nuns V, Cavalli M, et al. Comparative analysis between RQ-PCR and digital-droplet-PCR of immunoglobulinscell receptor gene rearrangements to manitor minimal residual disease in acute lymphoblastic leukaemia. Br J Haematol. 2016;174(4):541-549.

43. Bonvini P, Zn A, Alaggio R, Pawl B, Bisogno G, Rosolen A. High ALK mRA expression has a negative prognostic significance in rhabdomyosarcoma. Br J Cancer. 2013;109(12):3084-3091.

44. Jennings LJ, George D, Czech J, Xu M, Joseph L. Detection and quantification of BCR-ABL1 fusion transcripts by droplet digital PCR. J Mol Dian. 2014;16(2):174-179.

45. Iacobucci I, Lonetti A, Venturi C, et al. Use of a high sensitive nanofluidic array for the detection of rare copies of BCR-ABL1 transcript in patients with Philadelphia-positive acute lymphoblastic leukemia in complete response. Leak Res. 2014;38(5):581-585.

46. Wang WJ, Zheng CF, Lu Z, et al. Droplet digital PCR for BCR/ABL(P210) detection of chronic myeloid leukemia: A high sensitive method of the minimal residual disease and disease progression. Eur J Haematol. 2018; 101(3):291-296.

47. Huggett JF, Fry CA, Bees V, et al. The digital MIOE guidelines: minimum information for publication of quantitative digital PCR experiments. Chin Chem. 2013;59(6):892-902.

48. Huggett JF, Cower S, Fry CA. Considerations for digital PCR as an ecurate molecular diagnostic tool. Chin Chem. 2015;61(1):79-88.

haematological | 2020; 105(8)

2149 